\title{
Fiktive Realität: Basileios II. und Konstantinos VIII. in der „Chronographia” des Michael Psellos
}

\author{
RALPH-JOHANNES LILIE
}

In der Reihe der byzantinischen Kaiser gibt es kaum ein größeres Gegensatzpaar als die beiden Brüder Basileios II. (976-1025) und Konstantin VIII. (1025-1028). Während der eine als einer der größten byzantinischen Kaiser gilt, wenn nicht als der größte überhaupt, wird der andere als eitler Schwächling gesehen, mit dem der Niedergang des Reiches einsetzt, der in der Katastrophe von Mantzikert am 19. August 1071 gipfelt. Es mag ausreichen, die Charakterisierungen bei G. Ostrogorsky zu zitieren: „Als die bedeutendsten Kaiser von Byzanz nennt noch ein Schriftsteller des 13. Jahrhunderts Herakleios und Basileios II. Diese beiden Namen, in der Tat die größten der byzantinischen Geschichte, versinnbildlichen das heroische Zeitalter von Byzanz, das der eine von ihnen eröffnet und der andere abschließt.“ Zu Konstantin VIII. stellt derselbe Autor fest: „Wie er (Konstantin VIII.) im Laufe eines halben Jahrhunderts als Mitregent seinem großen Bruder untätig zur Seite stand, so hat er auch, im Greisenalter zur Herrschaft gelangt, den Staat mehr repräsentiert denn geleitet. Es fehlte ihm nicht an Begabung, wohl aber an Charakter und Verantwortungsgefühl. Die Regierungsgeschäfte überließ er anderen und verbrachte die Zeit bei Gastmählern und Hippodromspielen, die unter Basileios II. angesammelten Staatsschätze sorglos vergeudend.“'

Dieses Urteil hat im wesentlichen auch heute noch Bestand. Kritische Stimmen, die es hinterfragen, stellten bis vor kurzem eine kleine, kaum wahrgenommene Minderheit dar, deren Meinung auch heute noch nur begrenzt wahrgenommen wird. ${ }^{2}$ Das ist insofern überraschend, als bei etwas genauerem Hinsehen beide Charakterisierungen so gut wie ausschließlich auf einer einzigen Quelle

1 G. Ostrogorsky, Geschichte des byzantinischen Staates, dritte durchgearbeitete Aufl., (HdAW XII, Byzantinisches Handbuch 1,2), München 1963, 261. 265.

2 Zu Basileios II. cf. jetzt C. Holmes, Basil II and the Governance of Empire (976-1025) (Oxford Studies in Byzantium), Oxford 2005; ein Überblick über die frühere Literatur ebenda 16-29; zu Konstantin VIII. fehlt eine ausführliche Darstellung. Eine Skizze bietet K.-P. Todt, Herrscher im Schatten. Konstantin VIII. (960/61-1028), in: Thetis. Mannheimer Beiträge zur Klassischen Archäologie und Geschichte Griechenlands und Zyperns 7 (2000) 93-105. 
beruhen, der Chronographia des Michael Psellos. ${ }^{3}$ Für die Epoche um die Jahrtausendwende gibt es neben Psellos vor allem die Chronik des Johannes Skylitzes, die sich aber weit weniger als Psellos mit den Persönlichkeiten der beiden Brüder beschäftigt, dafür aber sonst erheblich detailreicher und genauer ist. ${ }^{4}$ Die etwas spätere Chronik des Johannes Zonaras schreibt sowohl Skylitzes als auch Psellos aus und übernimmt weitgehend dessen Charakterisierungen. ${ }^{5} \mathrm{Da}$ ausgerechnet Psellos in seiner Darstellung fast ohne Einschränkung gefolgt worden ist, verwundert, denn auf der anderen Seite galt der Politiker Psellos als in einer Weise moralisch verkommen, die in sich selbst schon wieder verzerrt erscheint. Zitieren wir noch einmal Ostrogorsky: „In seinem höchst fruchtbaren Wirken auf kulturellem Felde wie auch in seiner unheilvollen politischen Tätigkeit und abgründigen moralischen Verdorbenheit ist Psellos die markanteste Gestalt dieser Zeit [...] seine rhetorische Gabe (war) eine Waffe von schlechthin unwiderstehlicher Kraft. Psellos hat als Politiker von dieser Waffe reichen Gebrauch gemacht und sie oft in einer Weise mißbraucht, für die jedes Urteil zu milde erscheint.“6

Bedenkt man, da $\beta$ in der byzantinischen Historiographie gerade die rhetorische Ausgestaltung eines Geschichtswerkes eine besondere Rolle spielte, ja geradezu gefordert wurde, so ist doch überraschend, daß man die Chronographia des Psellos, zumindest in ihren frühen Abschnitten, gleichsam losgelöst von der Person offenbar als objektive Quelle angesehen und ihr weithin Glauben geschenkt hat, selbst dort, wo die inneren Widersprüche offensichtlich sind. Im folgenden soll daher versucht werden, einige Aspekte der Darstellung der beiden Kaiser Basileios II. und Konstantin VIII. in der Chronographia in Hinblick auf Tendenz und literarische Stilisierung zu analysieren. ${ }^{7}$

3 Michele Psello, Imperatori di Bisanzio (Cronografia), Volume I (Libri I-VI 75). Introduzione di D. del Corno, Testo critico a cura de S. Impellizeri, Commento di U. Criscuolo, Traduzione di S. Ronchey, Milano 1984; Basileios und Konstantin werden in den ersten beiden Büchern behandelt. Psellos wird im folgenden direkt im Text nach Buch und Kapitel zitiert, gegebenenfalls auch nach Zeilen; zu dem Bild Basileios' II. bei Psellos cf. Holmes, Basil II (s. Anm. 2), 29-35; allgemein zu der Chronographia cf. jetzt auch E. Pietsch, Die Chronographia des Michael Psellos. Kaisergeschichte, Autobiographie und Apologie (Serta graeca. Beiträge zur Erforschung griechischer Texte 20), Wiesbaden 2005. Die Autorin geht allerdings, so weit ich sehe, auf die hier behandelte Thematik nicht näher ein, sondern konzentriert sich vor allem auf die Selbstdarstellung des Psellos in der Chronographia.

4 Ioannis Scylitzae Synopsis historiarum, editio princeps, recensuit I. Thurn (CFHB V - Series Berolinensis), Berlin 1973.

5 Ioannis Zonarae epitome historiarum libri XIII-XVIII, ed. Th. Büttner-Wobst (CSHB), Bonn 1897.

6 Ostrogorsky (s. Anm. 1), 270f.

7 Cf. in diesem Sinn auch Holmes, Basil II (s. Anm. 2), 11; klarzustellen ist, daß es an dieser Stelle nicht um eine Neubewertung der Herrschaft Basileios' II. geht, sondern um die Darstellung seiner Person und derjenigen seines Bruders; zu der Einschätzung seiner Herrschaft cf. jetzt z. B. M. Angold, The Byzantine Empire 1025-1204, New York ${ }^{2} 1997$, Einleitung 1- 25 (eher kritisch); M. Whittow, The Making of Orthodox Byzantium, 600-1025, Basingsto- 


\section{Die Ausgangslage}

Psellos setzt ein mit dem Tod Johannes' I. Tzimiskes 976, den die Brüder Basileios und Konstantin als nahezu erwachsen erleben. Beide sind in seiner Schilderung jedoch völlig unterschiedlich: Basileios wachsam, klug und nachdenklich, dagegen Konstantin phlegmatisch, träge und allen Genüssen zugeneigt. Daher sei es nur natürlich gewesen, daß beide die Idee einer gemeinsamen Herrschaft aufgegeben hätten. Unter allgemeiner Zustimmung habe Basileios die Macht ausgeübt, während Konstantin nur im Titel mit ihm gleich gewesen sei. Dies sei eine weise Entscheidung zugunsten des Reiches gewesen (I, 2). Konstantin habe bei dieser Gelegenheit auf die meisten seiner Vorrechte verzichtet, was man ihm hoch anrechnen müsse, da er, juristisch gesehen, dieselben Rechte wie Basileios hätte beanspruchen können. Der Verzicht sei um so höher zu bewerten, als Konstantin zu dieser Zeit noch sehr jung gewesen sei, in einem Alter, wo die Begierde nach Macht ja noch groß sei (I, 2).

Diese Mitteilung steht fast am Anfang der Chronographia, an prominenter Stelle, und gibt somit gleichsam das Grundmotiv für beide Brüder vor: Basileios das Idealbild eines Herrschers, sein Bruder dagegen ein Weichling, der freiwillig auf die Bürde der Herrschaft verzichtet. Aber hat Konstantin überhaupt eine Wahl gehabt, wie Psellos es unterstellt? Basileios war der ältere, der von seinem Vater demgemäß auch zuerst zum Mitkaiser gekrönt wurde (22. April 960), während Konstantin erst zwei Jahre später zum Mitkaiser avancierte (wahrscheinlich am 30. März 962). Das hatte zur Folge, daß Basileios der nächste Hauptkaiser wurde und Konstantin von vorneherein auf eine zeremonielle Rolle beschränkt war, ohne daß es hierzu einer gemeinsamen Entscheidung der beiden Brüder bedurft hätte. Auch wenn beide, wie das üblich war, auf den offiziellen Dokumenten als gemeinsame Kaiser aufgeführt wurden, bedeutete das nicht, daß eine Samtherrschaft der beiden Brüder für Konstantin eine realistische oder auch nur legale Option gewesen wäre. Wenn es tatsächlich dazu gekommen wäre, wäre es das erste und einzige Mal in der gesamten byzantinischen Geschichte gewesen.

Psellos bewirkt durch diese Veränderung aber eine Gewichtsverschiebung zuungunsten Konstantins, auch wenn er es perfide in die Form eines Kompliments kleidet: Konstantin ist nicht gezwungenermaßen untätig und auf zeremonielle Aktivitäten beschränkt, sondern er wählt freiwillig dieses Leben des Müßiggangs. Er allein ist dafür verantwortlich, niemand sonst. Die Schuld fällt auf ihn selbst.

Im Gegensatz dazu steht Basileios, der für sich ganz bewußt ein Leben voll Anstrengung und Mühen wählt. Dabei hätte auch er laut Psellos durchaus die

ke/London 1996, 358-390 (eher positiv); Holmes (positiv); kritischer Überblick auch bei R.-J. Lilie, Byzanz. Das zweite Rom, Berlin 2003, 246-256. 
Wahl gehabt, ein leichteres Leben zu wählen, denn bis zu seiner Thronbesteigung habe er (genau wie Konstantin) ein leichtsinniges Leben geführt, mit häufigen Festen und zahlreichen Liebesabenteuern. Die Kombination von Jugend und unbegrenzter Macht hätte ihm alle Möglichkeiten gegeben, und er habe sie voll ausgekostet. Erst die Aufstände des Bardas Skleros und des Bardas Phokas hätten eine vollständige Veränderung in seinem Charakter und Verhalten bewirkt, so daß er zu dem „einsamen Heroen” wurde, als den Psellos ihn charakterisiert (I, 4. 18). Wir werden sehen, daß dieses Bild so mit Sicherheit nicht zutrifft.

\section{Konstantin in der Regierungszeit Basileios’ II.}

Konstantin tritt konsequenterweise während der Herrschaft seines Bruders bei Psellos so gut wie überhaupt nicht in Erscheinung. Die einzige bezeichnende Ausnahme ist die große Entscheidungsschlacht zwischen den kaiserlichen Truppen und dem Usurpator Bardas Phokas im Jahre 989 bei Abydos, bei der Bardas Phokas stirbt (I, 13-17). Das kaiserliche Heer wird von Basileios selbst geführt, dem gerade der erste Bart wuchs und der dabei war, die Art der Kriegsführung zu lernen. Fast verwundert notiert Psellos, daß auch Konstantin dabei war und in der Schlachtlinie stand, gepanzert und mit einem langen Speer bewaffnet. Die Ereignisse als solche sind bekannt. Phokas sah den Kaiser, sammelte einige Männer um sich und sprengte auf ihn los, um ihn zu töten und so den Krieg zu entscheiden. Dabei fiel er vom Pferd und blieb liegen. Psellos notiert einige Möglichkeiten, wie der Usurpator zu Fall gekommen sein könnte: Getroffen von einer Schleuder, ein Schwächeanfall aufgrund einer Unpäßlichkeit und schließlich Folge einer Vergiftung. Auch Konstantin soll sich gebrüstet haben, Phokas getötet zu haben. Aber das wird von Psellos leichthin abgetan. Für ihn ist Basileios selbst der Verantwortliche, der die Vergiftung des Phokas ins Werk gesetzt hatte (I, 16).

Bezeichnender aber ist die Behandlung Konstantins: Zunächst wird ihm ja vorgeworfen, das luxuriöse Leben eines Müßiggängers zu führen. Jetzt taucht er plötzlich doch in der Schlacht auf, aber natürlich nicht exponiert wie Basileios, sondern in der Schlachtreihe, praktisch wie ein gemeiner Soldat. Später wird dann konstatiert, daß er, freilich zusammen mit anderen, doch ein wenig vor der Schlachtreihe gestanden habe. Aber das wird gleich ins Lächerliche gezogen, indem Psellos ihm mehr oder weniger eitles Selbstlob unterstellt. Man könnte formulieren, daß Konstantin keine Chance bei Psellos hatte, was immer er auch tun mochte.

Sonst tritt Konstantin während der Regierungszeit seines Bruders nur noch einmal bei Psellos in Erscheinung. Nach der Entmachtung des Parakoimomenos 
Basileios haben sich der Charakter Basileios' II. und sein Auftreten völlig verändert. Er habe - neben anderem - alle Macht auf sich selbst vereinigt und alle anderen verachtet, auch seinen Bruder, dem er nur noch einige wenige Wachen zugestanden habe. Auch habe er ihm immer mehr an (kaiserlicher) Autorität entzogen. Konstantin blieben die Annehmlichkeiten des Landlebens, die Freuden von Bad und Jagd, während Basileios II. zum Krieg an die Grenzen des Reiches $\operatorname{zog}(\mathrm{I}, 22)^{8}$

Tatsächlich dürfte Konstantin während der Regierungszeit seines Bruders nur wenige ernsthafte Aufgaben übernommen haben. Indes war er nicht ganz so untätig, wie der Bericht des Psellos es nahelegt. Seine Teilnahme an der Schlacht bei Abydos erwähnt auch Psellos, verschweigt aber, daß Konstantin die Vorhut des kaiserlichen Heeres anführte, also durchaus eine herausgehobene Rolle innehatte. 9 Bei dem Ende der zweiten Revolte des Bardas Skleros soll Konstantin, wenn man dem arabischen Chronisten Ibn Yahya glauben will, eine vermittelnde Rolle gespielt und den Usurpator davon überzeugt haben, daß es besser sei, sich dem Kaiser zu unterwerfen. ${ }^{10} 995$ war er an dem Feldzug des Basileios gegen die Fatimiden beteiligt und soll einen Angriff auf Aleppo vorgeschlagen haben, was von Basileios abgelehnt wurde. ${ }^{11}$

$\mathrm{Daß}$ Konstantin während der Herrschaft seines Bruders nur eine eher dekorative Nebenrolle gespielt hat, ist nicht so sehr seinem angeblich schlaffen Charakter zuzuschreiben, sondern der Situation. Mitkaiser haben in Byzanz so gut wie niemals offen politische oder militärische Ämter ausgeübt, sondern sahen sich allein auf zeremonielle Funktionen beschränkt. ${ }^{12}$ Warum dies so war, braucht hier nicht untersucht zu werden. ${ }^{13}$ Das Faktum als solches ist unbestreitbar. Insofern kann

8 Psellos folgend: Zonaras (s. Anm. 5), XVII 7, 555,9-16, der den fehlenden Widerstand Konstantins mit dessen schlaffem Charakter begründet und hierbei die differenzierenden Bemerkungen des Psellos vereinfacht und zusammenzieht; ähnlich auch die wahrscheinlich im späten 11. Jahrhundert entstandene und (wohl fälschlich) unter dem Namen des Psellos laufende Zusammenstellung: Michaelis Pselli Historia Syntomos. Editio Princeps, rec., anglice vertit et commentario instruxit W. J. Aerts (CFHB XXX - Series Berolinensis), Berlin/New York 1990, 106,43-108,49. Auch hier ist der Einfluß der tendenziellen Darstellung der Chronographia unabweisbar.

9 Skylitzes (s. Anm. 4), Basileios 18, 337,6f.; Zonaras (s. Anm. 5), XVII 7, 553,4f. 13-17.

10 Histoire de Yahya-ibn-Sa 'ïd d'Antioche, ed./ transl. I. Kratchkovsky/A. Vasiliev, in: PO 23,3 (1932) 345-520, 427; ital. Übers.: Yahyā al-Anțakī, Cronache dell'Egitto fătimide e dell' impero bizantino 937-1033, traduzione dall'arabo, introduzione e cura di B. Pirone (Patrimonio Culturale Arabo Cristiano diretto da S. Kh. Samir S.J., Biblioteca del Vicino Oriente 3), Milano 1988,10:161, 198.

11 W. A. Farag, The Aleppo Question: A Byzantine-Fatimid Conflict of Interest in Northern Syria in the Later Tenth Century, in: BMGS 14 (1990) 44-60, 53.

12 Die einzige wirklich relevante Ausnahme ist wohl Manuel II. Palaiologos gewesen, der allerdings in der Spätzeit des Reiches, im 14./15. Jahrhundert, tätig war. In der mittelbyzantinischen Zeit ist mir kein einziges Beispiel bekannt.

13 Denkbar wäre, daß die Kaiser eine zu starke Stellung des Mitkaisers und damit eine Bedrohung 
man diese fehlende Aktivität Konstantins auch nicht zur Beurteilung seines Charakters heranziehen, da er keine Chance hatte, eine aktivere Rolle zu spielen.

\section{Die Regierungszeit Basileios’ II.}

Die gegensätzliche Charakterisierung der beiden Brüder zeigt sich sowohl im direkten Vergleich als auch in der jeweiligen Einzeldarstellung. Basileios II. wird durchgehend als aktive Persönlichkeit dargestellt, die zugunsten des Reiches auch persönliche Opfer auf sich nimmt. Das beginnt bereits mit dem Herrschaftsantritt nach dem Tod Johannes' I. Tzimiskes, als Basileios laut Psellos in voller Einsicht seiner eigenen Unzulänglichkeit die Ausübung der Regierung seinem Onkel (tatsächlich Großonkel), dem Parakoimomenos Basileios, überließ. Basileios habe alles dem Parakoimomenos anvertraut und ihm gleichsam nur zugesehen, dabei aber an seinem Beispiel gelernt und seine Art und Weise nachgeahmt. So hätte der Parakoimomenos, der voller Liebe für seinen Schützling war, die Regierung geleitet, sei dabei aber von dem jungen Kaiser in jeder Weise unterstützt worden (I, 3).

Es folgt die schon genannte Wandlung im Charakter Basileios' II., die laut Psellos zum Teil schon mit der Alleinherrschaft einsetzt, zum Teil erst durch die Usurpationen des Bardas Skleros und des Bardas Phokas bewirkt wurde (I, 4. 18). Diese Rebellionen bilden den Kern der Darstellung. Sie nehmen den zentralen Platz in dem Basileios gewidmeten Buch ein. Bis zu ihnen zeigt Basileios zwar schon Zeichen seiner charakterlichen Festigkeit, aber erst in den „Stahlgewittern” dieser Kämpfe wird er zu der herausragenden Persönlichkeit, als die er seinen Zeitgenossen im Gedächtnis geblieben sei.

Zunächst rebellierte Skleros, der durch Bardas Phokas niedergeworfen wurde und in das Kalifat floh (I, 5-9). Aber Bardas Phokas fühlte trotz hoher Ehrungen seine Verdienste nicht genügend gewürdigt und rebellierte seinerseits. Er zog gegen Konstantinopel, kam aber schließlich bei Abydos zu Tode (I, 10-17). Nach dem Ende des Bardas Phokas entledigte Basileios II. sich des Parakoimomenos Basileios, wie Psellos andeutet, auf eine eher undankbare und brutale Art und Weise. Zugleich wurde er jetzt immer herrischer. Er konzentrierte die gesamte Macht auf sich selbst. In diese Periode fallen auch die geschilderten Maßnahmen gegen seinen Bruder Konstantin (I, 19-22).

Basileios wollte nun gegen die umwohnenden Barbaren ziehen, wurde davon aber durch die zweite Rebellion des Bardas Skleros abgehalten, bis dieser sich

ihrer eigenen Position fürchteten. Ebenso ließe sich annehmen, daß man den Thronfolger keiner Gefahr aussetzen wollte, um den Fortbestand der Dynastie nicht zu gefährden. Aber das sind nur Spekulationen. 
schließlich ergab, u. a. gegen die Zusicherung, den ersten Platz hinter dem Kaiser selbst einnehmen zu dürfen - was bedeutet hätte, daß er noch vor Konstantin rangiert hätte (I, 23-26), und was daher real gar nicht möglich gewesen wäre, denn dann hätte Basileios Bardas Skleros zum Mitkaiser, der im Rang vor seinem schon lange gekrönten Bruder stand, erheben müssen, was unvorstellbar gewesen wäre. ${ }^{14}$

Es folgt eine Schilderung der autokratischen Art, mit der Basileios II. von nun an regierte, der nicht nur die Barbaren, sondern auch seine eigenen Untertanen unterjochte, einen großen Staatsschatz anhäufte, in allen Bereichen informiert war und insbesondere seine Armee zu einer kampfkräftigen Einheit schulte, die er persönlich ins Feld führte (I, 30-33). Die Darstellung schließt mit einer nochmaligen Schilderung des eisernen Charakters dieses Kaisers (I, 34).

Wenn wir die - nicht nachprüfbaren - Charakterschilderungen einmal auslassen, so ist die Darstellung des Psellos zwar in sich stimmig, aber nichtsdestoweniger in den entscheidenden Punkten falsch. Tatsächlich hatte Basileios II. im Jahre 976 nichts zu entscheiden, sondern war vollkommen von dem Parakoimomenos abhängig, dem er den Thron verdankte. Insofern kann man auch nicht davon sprechen, daß er aus Einsicht in die eigene Unerfahrenheit hier freiwillig auf die Macht verzichtet hätte. Ebenso ist falsch, daß Basileios II. sich sozusagen bewußt an dem Beispiel des Parakoimomenos geschult hätte. Wir wissen aus einer arabischen Quelle, die eine Gesandtschaft im Jahre 983 nach Konstantinopel schildert, daß Basileios die Zurücksetzung hinter den Parakoimomenos nur mit Widerwillen ertrug und versuchte, seine Position ihm gegenüber mit allen Mitteln zu verbessern. Wenn man von dieser Quelle ausgeht, dann war die reale Machtlosigkeit für die „Charakterbildung” Basileios' II. wahrscheinlich entscheidender als die Usurpationen des Bardas Skleros und des Bardas Phokas. ${ }^{15}$

Wichtiger noch sind die chronologischen Unstimmigkeiten, die nicht auf Fehlinformationen beruhen können, sondern bewußte Umstellungen sind. Die Usurpation des Bardas Skleros (976-979) ist zwar korrekt eingeordnet, aber Psellos schließt direkt die Usurpation des Bardas Phokas an, die tatsächlich erst 986989 stattfand, und schiebt sie damit vor die Entmachtung des Parakoimomenos (985-986). Damit tritt eine völlige Gewichtsverschiebung ein: Die beiden Usurpationen werden zu den großen Bewährungsproben des Kaisers, aus denen er gleichsam gestählt hervorgeht. Danach ist er so weit gereift, daß er die Bevor-

14 Skylitzes (s. Anm. 4), Basileios 19, 338,45-339,63 ist hier genauer, wenn er schreibt, daß Bardas Skleros zum Kouropalates ernannt worden sei. Aber die Formulierung in der Chronographia ist bezeichnend für den Stellenwert Konstantins bei Psellos.

15 H. F. Amedroz, An Embassy from Baghdad to the Emperor Basil II., in: JRAS 1914, 915-942 (engl. Übers.: 919-931; arab. Text: 933-942); cf. auch A. Beihammer, Der harte Sturz des Bardas Skleros. Eine Fallstudie zu zwischenstaatlicher Kommunikation und Konfliktführung in der byzantinisch-arabischen Diplomatie des 10. Jahrhunderts, in: RHM 45 (2003) 21-57. 
mundung durch den Parakoimomenos abschütteln kann. Es folgt die zweite Erhebung des Bardas Skleros (tatsächlich 989) als verzögerndes Moment, ehe Basileios sich den äußeren Feinden widmen kann.

Tatsächlich haben die Ereignisse sich wesentlich länger und mit größeren Unterbrechungen hingezogen, als Psellos es hinstellt. Zunächst gab es die Revolte des Skleros (976-979), dann folgte eine längere Periode, in der Basileios II. offenbar vorsichtig versuchte, die übermächtige Stellung des Parakoimomenos zu erschüttern (979-985). Das gelang erst 985/86, als Basileios seinen Großonkel absetzte und verbannte. Anschließend (im Sommer 986) brach Basileios gegen die Bulgaren auf, erlitt aber eine schwere Niederlage. Erst diese beiden Ereignisse dürften die Erhebung des Bardas Phokas ausgelöst haben, die Basileios in größte Bedrängnis brachte und aus der ihn nur das Bündnis mit den Varägern aus Kiev rettete, die dafür u. a. mit der Schwester des Kaisers „bezahlt” wurden, die den Großfürsten von Kiev heiraten mußte.

Der Unterschied zur Schilderung des Psellos ist offensichtlich. Bei Psellos ist es Basileios selbst, der den Gang der Ereignisse bestimmt und diejenigen, die seine Herrschaft in Frage stellen, unterwirft. Selbst die - zeitweilige - Unterordnung unter den Parakoimomenos ist seine eigene, freiwillige Entscheidung. Man könnte aber auch durchaus den gegenteiligen Standpunkt einnehmen: Erst die Entmachtung des Parakoimomenos durch den Kaiser und die schwere Niederlage, die das byzantinische Heer unter der persönlichen Führung des Basileios erlitt, führten zu der Revolte des Bardas Phokas. Seine schwierige Lage wäre demnach seine eigene Schuld gewesen. Jedoch hätte dies nicht in die Absicht des Psellos gepaßt, Basileios zum Helden zu erheben, den er den Kaisern seiner eigenen Zeit als Beispiel und Gegenbild gegenüberstellen konnte.

\section{Die Regierungszeit Konstantins VIII.}

Gegenüber der glanzvollen „Alleinherrschaft” (im Wortsinn) Basileios' II. weist die Regierungstätigkeit seines Bruders in der Darstellung des Psellos alle Anzeichen eines schwachen Kaisers auf. Sie entspricht damit freilich voll und ganz der Persönlichkeit Konstantins VIII., der eben nicht das Format seines Bruders aufwies. Konkrete Handlungen werden dabei von Psellos mit einer einzigen Ausnahme nicht genannt, sondern nur allgemeine Werturteile abgegeben. So soll Konstantin, der ohne jeden Widerstand seine zweite Herrschaftsperiode angetreten habe, den Staatsschatz verschwendet haben (II, 1). Von den Nachbarn des Reiches soll er Frieden durch Tributzahlungen erkauft haben (II, 2). Er kümmerte sich nicht um den Staat, sondern überließ alles seinen Vertrauten, während er selbst allenfalls einige protokollarische Pflichten übernahm, wie etwa Audienzen 
bei auswärtigen Gesandtschaften (II, 6). Theater, Wagenrennen und dem Würfelspiel war er so verfallen, daß er darüber die Angelegenheiten des Staates vergaß (II, 9). Dafür war er grausam gegenüber seinen Untertanen, die er gerne foltern und vor allem blenden ließ (II, 2).

Diese eher allgemeinen Angaben lassen sich naturgemäß im einzelnen kaum überprüfen. $\mathrm{Da} ß$ Konstantin die Feinde des Reiches durch Tributzahlungen ruhiggestellt habe, findet in den anderen Quellen seiner Regierungszeit keine Stütze. Ein regelrechter Vertrag ist nur mit den Fatimiden bekannt, mit denen aber auch Basileios II. schon Verträge geschlossen hatte. Konstantin stellte hier praktisch nur den früheren Zustand wieder her. ${ }^{16}$

Geschickt verzerrt sind auch die Aussagen des Psellos über die angebliche Grausamkeit Konstantins. In seiner Darstellung war Konstantin ja ohne jeden Widerstand auf den Thron gekommen, von etwaigen Verschwörungen ist nirgendwo die Rede, so daß die Grausamkeiten des Kaisers völlig unmotiviert wirken und damit allein auf den schlechten Charakter des Kaisers zurückzuführen sind. Tatsächlich überliefert auch Skylitzes, der Konstantin gleichfalls ablehnend gegenübersteht, daß viele Gegner Konstantins geblendet worden seien, wobei er im Unterschied zu Psellos auch zahlreiche Namen nennt. Doch geht aus der Chronik des Skylitzes wie auch aus dem Bericht des arabischen Autors Yahya von Antiochia hervor, daß es einige Verschwörungen gegeben hatte, so daß die angebliche Grausamkeit Konstantins zumindest relativiert werden muß. ${ }^{17}$

Wohl nicht zu bestreiten ist die skandalöse Regelung der Nachfolge des Kaisers mit der - bei Psellos nicht erklärlichen - Auswahl des verheirateten Romanos Argyros als Gatten Zoes, der zweiten Tochter Konstantins (II, 10). Aber auch hier könnte man einwenden, daß dieser offenbar unverhofft starb, so daß die Auswahl des Nachfolgers überstürzt erfolgte. Psellos verschweigt aber, daß Romanos III. ein entfernter Verwandter des Kaiserhauses war, so daß seine Wahl nicht völlig grundlos erfolgte. Außerdem könnte man hier gleichfalls Basileios II. in die Verantwortung ziehen, der ja gleichfalls keine über seinen Bruder hinausgehende Nachfolgeregelung getroffen hatte. Jedoch wird Basileios von jeder Verantwortung für die Töchter Konstantins entlastet, indem Psellos zwar einerseits die Liebe des Basileios für seine Nichten hervorhebt, aber andererseits erklärt, daß die ganze Erziehung Konstantin überlassen geblieben sei, da Basileios das Reich für

16 Regesten der Kaiserurkunden des Oströmischen Reiches von 565-1453, bearbeitet von F. Dö1ger, 2. Teil: Regesten von 1025-1204. Zweite erweiterte und verbesserte Auflage, bearbeitet von P. Wirth, mit Nachträgen zu Regesten Faszikel 3 (Corpus der griechischen Urkunden des Mittelalters und der Neueren Zeit, Reihe A: Regesten, Abt. I: Regesten der Kaiserurkunden des Oströmischen Reiches), München 1995, Nr. 824 von 1027 a. d.

17 Einen Überblick über die Verschwörungen und Aufstände zwischen 976 und 1028 gibt J.-C. Cheynet, Pouvoir et Contestations à Byzance (963-1210) (Byzantina Sorbonensia 9), Paris 1990, 27-41 (Nr. 11-30). 
seinen Bruder gehütet habe. Hier wird sogar die Feldherrntätigkeit des Basileios gegen Konstantin gekehrt (II, 4).

Ihre Schärfe erhält die Darstellung des Psellos aber nicht so sehr durch die Darstellung der Versäumnisse Konstantins VIII. als Kaiser, sondern durch seine Charakterisierung, bei der Psellos alle Register zieht und auch vor direkten inneren Widersprüchen nicht zurückschreckt. So legt er in einem Kapitel großen Wert darauf, daß Konstantin infolge seiner Genußsucht, insbesondere Essen und Sex, so heftig unter Arthritis gelitten habe, daß er sich während seiner Regierungszeit überhaupt nicht mehr zu Fuß, sondern nur noch zu Pferd fortbewegt habe (II, 7). Im folgenden Kapitel wird dann die Leidenschaft des Kaisers für Theater, Wagenrennen und die Jagd hervorgehoben, und Psellos erklärt, daß der Kaiser die Gymnopaidiai wieder eingeführt habe. Er habe sich aber nicht auf die Zuschauerrolle beschränkt, sondern sei selbst als Aktiver hervorgetreten und habe die anderen Teilnehmer aufgefordert, sich Mühe zu geben, damit sein Sieg umso ehrenvoller sein würde (II, 8). Bei diesen Gymnopaidiai handelt es sich wahrscheinlich um eine Reminiszenz an die bekannten Gymnopaidiai aus Sparta, eine Art von Tanzund Gymnastikspielen. ${ }^{18}$ Wie ein alter Mann von über 70 Jahren, der sich erklärtermaßen kaum noch auf den eigenen Füßen halten kann, andererseits als Wettkämpfer an einem Tanz- und Gymnastikwettkampf teilzunehmen in der Lage sein soll, erklärt Psellos nicht. Es ist auch nicht nötig, denn es geht ihm nicht um die Tatsachen als solche, sondern darum, hier Assoziationen zu den anderen Kaisern herzustellen, die in lächerlicher Weise öffentlich auftraten, wie etwa Nero in römischer oder auch Michael III. in byzantinischer Zeit.

Als weiteres Stilmittel für die negative Charakterisierung Konstantins dient der Vergleich mit seinem großen Bruder Basileios II., demgegenüber Konstantin in praktisch allen Belangen zum negativen Spiegelbild stilisiert wird, sei es im ausgesprochenen Vergleich oder eher indirekt, wobei Psellos darauf vertrauen kann, daß dem Leser das Bild des Basileios noch gegenwärtig ist.

Diese Gegensätze treten sowohl in Äußerlichkeiten zutage wie auch in der Art und Weise, wie beide ihre Herrschaft ausüben:

Konstantin ist ausgesprochen groß (II, 7), während Basileios kleiner als der Durchschnitt ist (I, 36). Konstantin ist ängstlich (II, 2), Basileios scheut vor keinem Krieg zurück (I 22). Konstantin wird als phlegmatisch und träge geschildert (I, 2), während Basileios aktiv und aufmerksam über die Belange des Reiches wacht (II, 4). Basileios ist voller Selbstbeherrschung, Konstantin hingegen braust schnell auf und neigt zu spontanen, unüberlegten Handlungen (II, 2). Demgegen-

18 In der älteren Edition von É. Renauld (Michel Psellos, Chronographie I, Paris 1967, II 8,3-8 [29]) heißt es noch Gymnopodia. Impellizeri schließt sich jedoch einer älteren Korrektur von P. Maas an. Aber auch wenn Gymnopodia zutreffend sein sollte, so würden auch sie eine körperliche Aktivität bezeichnen. Zu dem Gebrauch des Wortes in byzantinischer Zeit cf. LBG 1, 332. 
über ist Basileios beständig. Wenn er einmal eine Meinung gefaßt hat, bleibt er bei ihr (I, 4). Konstantin hingegen bereut seine vorschnellen Handlungen fast sofort. Konstantin ist verweichlicht und liebt jeden Luxus (II, 1). Basileios dagegen verachtet Luxus und lebt auch als Kaiser geradezu spartanisch (I, 22). Dies zeigt sich auch im persönlichen Gehabe: Basileios verabscheut jedes rhetorische Gepränge. Wenn er redet, dann kurz und abgehackt, sozusagen militärisch (I, 22). Konstantin hingegen verfügt über eine fließende Rede, ja geradezu über einen Redeschwall, der jeden Sekretär überfordert, so daß seine Schreiber sich stenographische Kürzel ausdenken müssen, um mit ihm mitzuhalten (II, 7). Man könnte auch sagen: Für Psellos ist Konstantin ein Schwätzer.

Dies sind eher persönliche Charaktereigenschaften. Aber auch in ihrer kaiserlichen Herrschaft sind die beiden Brüder völlige Gegensätze: Konstantin verläßt sich auf andere, die die Arbeit für ihn machen, besonders auf die Eunuchen aus seinem Gefolge, auch wenn sie für diese Arbeit nur bedingt geeignet sind (II, 3). Basileios herrscht allein und traut niemandem (I, 18). Basileios ist sparsam und häuft während seiner Regierungszeit einen riesigen Staatsschatz an (I, 31), der anschließend von dem Verschwender Konstantin vergeudet wird (II, 3. 9). Basileios unterwirft die Nachbarn des Reiches und ist ununterbrochen für das Reich aktiv (I, 22. 32), während Konstantin zu feige zur militärischen Auseinandersetzung ist und die Feinde durch Tributzahlungen ruhigstellt (II, 2). Im Endeffekt läßt der Unterschied sich an zwei Bemerkungen festmachen: Basileios ist nicht nur ein erfolgreicher Feldherr, sondern er führt Kriege, um zu gewinnen. Er hört nicht im Winter auf, sondern kämpft bis zum Sieg, und er kennt jeden Aspekt des Soldatenlebens (I, 32-33). In einem kaum stärker denkbaren Gegensatz hierzu steht die Feststellung, daß Konstantin ein unübertroffener Experte für die Herstellung von wohlschmeckenden Soßen ist (II 7). Aber eigentlich reicht schon die abschließende Bemerkung des Psellos, um Konstantin hinreichend zu charakterisieren: Nachdem Psellos ausführlich auf die Spielleidenschaft Konstantins eingegangen ist, zu deren Gunsten der Kaiser alles, auch ernsthafte Staatsgeschäfte, hintangestellt habe, subsumiert er seine Regierungszeit folgendermaßen: „Während er so seine Herrschaft im Spiel verbrachte, wurde er vom Tod überrascht" (II, 9,7f.). Was für ein Gegensatz zu Basileios, der Tag und Nacht darüber nachdachte, wie er sein Reich vor Unheil bewahren könnte!

Es ist natürlich nicht ausgeschlossen, daß einige, wenn nicht die meisten der von Psellos übermittelten Nachrichten zutreffend sind. Aber wo man feststellen kann, daß Psellos seine Vorlagen modifiziert hat oder wo innere Widersprüche in seiner Darstellung auftreten, weisen sie eigentlich immer in dieselbe Richtung: Basileios wird zum einsamen und großen Staatsmann und Kaiser stilisiert, während Konstantin das genaue Ebenbild darstellt: ein schwacher Kaiser, nicht interessiert am Wohlergehen des Reiches, abhängig von seiner Umgebung und nur 
auf das eigene Vergnügen bedacht. Dies läßt vermuten, daß auch die anderen möglicherweise im einzelnen ja zutreffenden - Charaktereigenschaften beider in die jeweilige Richtung hin übersteigert worden sind.

Die Vermutung liegt nahe, daß der Eigenwert Konstantins hierbei für Psellos geringer wiegt als derjenige Basileios' II. Basileios ist für Psellos das Gegenbild der Kaiser seiner eigenen Zeit. Er steht für die goldene Epoche der byzantinischen Geschichte, als Byzanz noch die große Macht war, furchterregend für die Nachbarn und unüberwindbar. Dies gilt aber nicht nur nach außen, sondern ebenso für die inneren Verhältnisse des Reiches. Bezeichnend für den Unterschied ist eine eher nebensächliche Bemerkung: Nachdem Psellos die kunstlose, allem rhetorischen Gepränge abholde Sprache des Basileios notiert hat, fragt er sich, wieso gerade unter so einem Kaiser die Redekunst in besonderer Blüte stand, und er findet als Antwort, daß es den damaligen Literaten eben um die Sache als solche gegangen sei, nicht darum, mit ihren Fähigkeiten Gut und Geld zu gewinnen (I, 29). Indirekt auch hierfür verantwortlich und zugleich beispielgebend ist der Kaiser Basileios, der die Belange des Reiches vor alles andere stellte, während die späteren Kaiser durch ihr eigensüchtiges Verhalten, ihre Schwachheit und Unfähigkeit das Elend des Reiches zur Zeit des Psellos verursachten. Konstantin VIII. ist der erste dieser Epigonen und daher in gewisser Weise als Prototyp für die nachfolgenden Herrscher gestaltet. An der konkreten Realität ihrer jeweiligen Herrschaft war Psellos weniger interessiert als daran, hier beispielhaft die Verhältnisse seiner eigenen Zeit zu kritisieren. Da dies direkt nur unter Schwierigkeiten möglich gewesen wäre, griff Psellos zu dem Mittel des Vergleichs und charakterisierte Basileios II. als Idealbild eines Kaisers, während sein Bruder Konstantin VIII. umgekehrt zum Gegenteil stilisiert wurde. Für seine Zeitgenossen dürften die vielfachen Anspielungen noch viel klarer gewesen sein als für den heutigen Leser, der die Verhältnisse im Byzanz des 11. Jahrhunderts und insbesondere die damals handelnden Personen fast nur noch im Zerrspiegel der Darstellung des Psellos kennt. 